\title{
The Effect of Biologically Active Substances on Bacillus subtillis Growth and Functional activity
}

\section{Lazarev Sergey Alexandrovich*, Mikhailova Natalia Alexandrovna, and Voevodin Dmitriy Anatolievich}

Federal State Budgetary Scientific Institution «l. Mechnikov Research Institute of Vaccines and Sera», Moscow, Russia

\section{ORCID:}

Lazarev Sergey Alexandrovich - https://orcid.org/0000-0003-3206-6015

Mikhailova Natalia Alexandrovna - https://orcid.org/0000-0002-6652-2093

Voevodin Dmitriy Anatolievich - https://orcid.org/0000-0002-2595-9168

Corresponding Author: Lazarev Sergey Alexandrovich; email: lazarevsr1@gmail.com

Published 13 January 2022

Publishing services provided by Knowledge E

(c) Lazarev Sergey Alexandrovich et al. This article is distributed under the terms of the Commons Attribution License, which permits unrestricted use and redistribution provided that the original author and source are credited.

Selection and Peer-review under the responsibility of the 8th Scientific and Practical Conference Conference Committee.
Abstract. For the most part the probiotics development is aimed at finding new effective strains and / or formation of original microbial consortia. It is believed that for the implementation of a therapeutic effect, it is enough for strains to reach in the host's body. However, the experience in practical use of such drugs in most cases revealed their insufficient therapeutic activity. In the last decade, the number of reports on the effect of the microorganisms habitat conditions as a resolving factor on the manifestation of one or another functional activity by them has increased. Herewith, it was suggested about the key role of peptide (cytokine)-regulation in the implementation of this process. Many regulators of protein-peptide nature are hydrolysis products. This study was carried preliminary assessment of industrial hydrolysates as a source of regulatory substances that affect the growth and functional properties of the $B$. subtilis. The hydrolysates used effect on the $B$. subtilis growth was assessed by the bacterial growth curve. The hydrolysates effect on the $B$. subtilis functional activity was assessed by their ability to produce antibacterial metabolites. As a result of the study, the change nature in growth indicators testified that industrial hydrolysates, in addition to nitrogenous nutrients, contain a number of biologically active substances, with can both suppress and stimulate the $B$. subtilis growth. The dependence of the $B$. subtilis antibacterial metabolites formation on hydrolysates and their heat treatment is shown. The presence of both thermolabile and thermostable biologically active substances was noted.

Keywords: Bacillus subtilis, biologically active substances, antibacterial metabolites, hydrolysates, probiotics

\section{Introduction}

The problem of antibiotic resistance of infectious diseases pathogens has directed researchers to the creation of alternative therapies - probiotics. Their action mechanism is based on the antagonistic properties of probiotic strains that produce biologically active metabolites that inhibit the growth and reproduction of pathogenic flora and 
stimulate the microbiota, providing permanent colonization resistance in the host's body [1].

For the most part the development of such drugs is aimed at finding new effective strains of microorganisms and / or formation of original microbial consortia. Such approach is due to the dominant concepts of genetic determinism (constitutiveness, constancy) in the manifestation of the functional properties of microorganisms. When creating new drugs of probiotics, at present, proceed from the idea that for the implementation of a therapeutic effect, it is enough for microorganisms to reach in the host's body. However, the experience in practical use of such probiotic drugs in most cases revealed their insufficient therapeutic activity and caused doctors to disappointment in probiotic therapy. These circumstances lead to the need for a critical reassessment of the position on the dominant role of genetic determinism in the manifestation of the functional properties of microorganisms.

In the last decade, the number of reports on the effect of the microorganisms habitat conditions as a resolving factor on the manifestation of one or another functional activity by them has increased. On the model of pro / eukaryotic heterogeneous systems is shown role of the host organism, its signal (command) effects on the manifestations of the functional activity of saprophytic microflora. Herewith, it was suggested about the key role of peptide (cytokine) regulation in the implementation of this process [2], as a particular reflection of a single, general biological mechanism of peptide-regulation of gene expression and proteins synthesis in nature [3]. Many regulators of protein-peptide nature originate from precursor molecules and are products of hydrolysis.

Earlier, when studying probiotic strains of Bacillus spp. was revealed that the synthesis of antibacterial metabolites depended on the protein hydrolyzate used as the basis of the culture medium on which the studied cultures were grown. When peptone was used, antagonistic activity was weak or absent. Replacement of peptone for tryptone in the culture medium qualitatively increased the antagonistic activity in the studied strains [4]. We suppose that the observed result is due to a complex of regulatory biologically active substance formed during obtaining of the tryptone and initiating the functional (antagonistic) activity of Bacillus spp.

This study was carried out with the aim of testing the assumptions made and preliminary assessment of industrial hydrolysates as a source of biologically active regulatory substances that affect the growth and functional properties of the Bacillus subtilis probiotic strain. 


\section{Materials and Methods}

\subsection{Biologically active regulatory substances.}

As a source of biologically active substances (protein-peptide compounds), solutions of industrial hydrolysates were used: tryptone (TR) (BD Bacto Tryptone, France) or pancreatic hydrolysate (PHL) (State Research Center for Applied Microbiology and Biotechnology, Obolensk, Russia). To assess the thermal stability of biologically active substances of hydrolysates, part of the prepared culture medium was sterilized by membrane filtration through filters with a pore size of $0.2 \mu \mathrm{m}$ (Millipore, France), part by autoclaving.

\subsection{Strains}

In the study used strain $B$. subtilis $3[$ and test-strains (to test antibacterial activity) S. aureus 29213, S. aureus FDA-209P, P. aeruginosa 103, E. coli [प] 25922 from The Mechnikov NIIVS collections.

\subsection{Method for assessing the effect of hydrolysates, their concen- trations and thermolability, on the growth $B$. subtilis.}

In the wells of a 96-well sterile plate, containing $50 \mu$ of nutrient broth, $50 \mu$ of TR solution or PHL sterilized by different methods were added in serial two-fold dilution. Then, $50 \mu$ of bacterial cells suspension were added to them B. subtilis ( $10^{4} \mathrm{CFU} / \mathrm{ml}$ ). The maximum working concentration of TR or PHL was $30 \mathrm{~g} / \mathrm{I}$. The growth properties were assessed using the «Microbe-2» software on a Multiskan-Ascent plate photometer base (Termo-Labsystems, Finland) equipped with a thermostat and a shaker.

\subsection{Method for assessing the effect of hydrolysates on the antibacterial activity by $B$. subtilis metabolites.}

B. subtilis was cultivated in potato-glucose broth (PGB), with the addition of TR to a concentration $3 \mathrm{~g} / \mathrm{l}$, at a temperature of $37^{\circ} \mathrm{C}$, for 24 hours, under aeration conditions. Supernatants containing $B$. subtilis metabolites were separated by centrifugation followed by sterilization through membrane filters with a pore size of $0.2 \mu \mathrm{m}$ (Millipore, France). On their basis, bacterial suspensions of test-strains $\left(10^{4} \mathrm{CFU} / \mathrm{ml}\right)$ were made 
Fig.1a. B.subtilis growth with TR

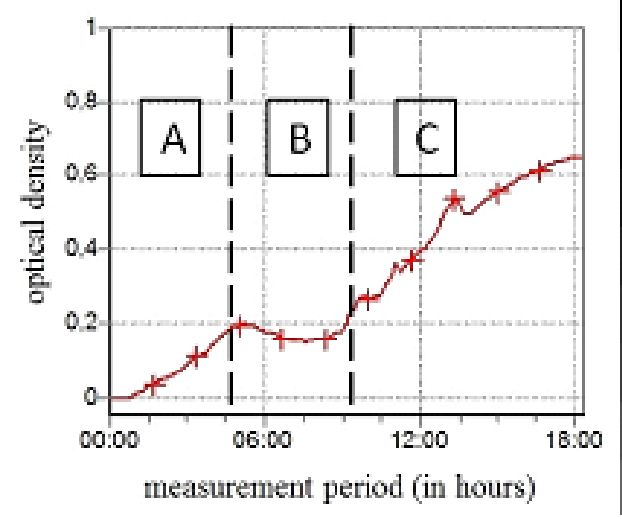

Fig.1b. B.subrilis growth with PHL

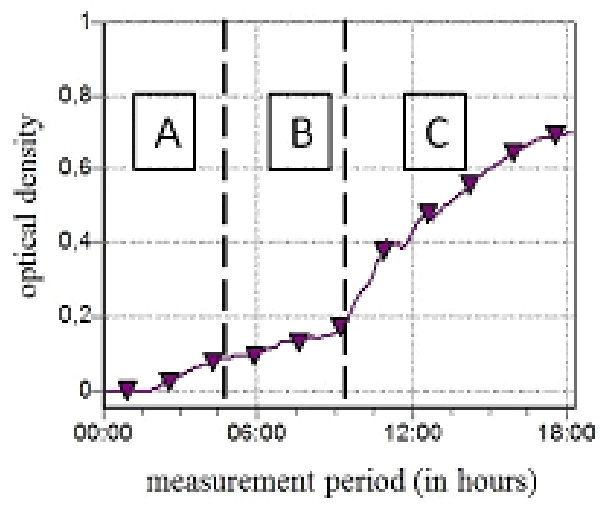

Figure 1: The effect of TR and PHL solutions sterilized by filtration on the $B$. subtilis growth properties.

and cultured at $37{ }^{\circ} \mathrm{C}$. After 24 hours, the optical density was measured on a spectrophotometer at a wavelength of $600 \mathrm{~nm}$. Bacterial suspensions of test-strains added to the nutrient broth were used as a control. The results of calculating the optical density were processed in Excel, determining the arithmetic mean values for each test-strain. On the basis of arithmetic mean data diagrams were built.

\section{Results and Discussion}

The character of the curves reflecting the $B$. subtilis growth processes is contradictory, which, in our opinion, indicates the diversity of the evaluated hydrolysates effects on the processes occurring in bacterial communities. Depending on the cultivation time, the character of the hydrolysates effect changed up to the exact opposite, which was especially pronounced when using TR solutions, sterilized by filtration. In accordance with the obtained curve in the culture growth process, three stages were distinguished: the stage of culture active growth $(A)$, in the range of cultivation 5-10 hours. Suppression stage (B), with a drop in growth activity by $30-40 \%$. And the stage of growth processes reactivation (C) (Fig. 1a). When PHL was added to the culture medium, there was no suppression of growth activity in stage B (Fig. 1b).

Stage "A" manifested itself in all cases, did not depend on the composition of the culture medium and sterilization conditions. Only the dependence on the concentration of the hydrolyzate in the culture medium was noted. Therefore, stage "A" can be considered as a process through the growth potential of the microorganisms themselves and the nutritional characteristics of the culture medium. The manifestation of stages "B" and "C" depended not only on the concentration of the agent in the medium, but on the method of hydrolysis and the method of sterilization (Fig. 2). 
Fig.2a. B.subtilis growth with TR

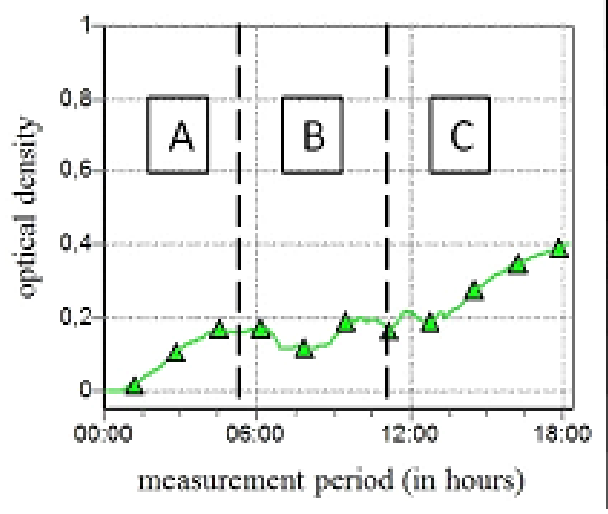

Fig.2b. B.subtilis growth with PHL

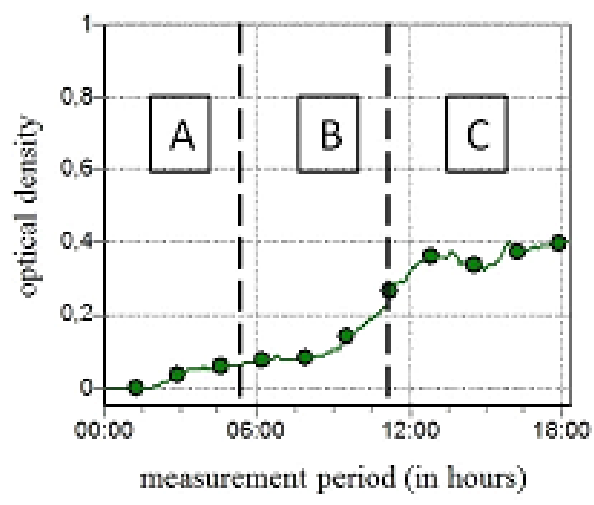

Figure 2: The effect of TR and PHL solutions sterilized by autoclaving on the $B$. subtilis growth properties.

The stage of growth processes reactivation "C" is due to the presence of thermolabile regulatory agents and is completely lost during temperature sterilization. In our opinion, these circumstances indicate on presence of biologically active substances in a composition of the used hydrolysates. And also on the formation in one hydrolyzate a number of biologically active agents with different functional focus.

The use of hydrolysates as a source of biologically active substances increases the requirements for quality control on the final product. $\mathrm{PHL}$, according to our data, can serve as a source of factors for stimulating the $B$. subtilis growth and contribute to the accumulation of bacterial mass. However, it turned out that this property of the $\mathrm{PHL}$ is not stable. We believe that this is due to insufficiently quenched proteolytic activity and hydrolysis resumption during preparation of solution. Taking into account the technological imperfection of this PHL, we refused to further work with it.

When using the regulatory properties of hydrolysates in the work, it is necessary to take into account that the resulting biologically active substances can be thermolabile with a loss of regulatory characteristics during routine thermal sterilization of culture media. The use of thermal sterilization was lead to the inactivation of the stimulating activity growth both PHL and TR. On the one hand, this circumstance allows the use of temperature exposure as a cheap and accessible method of fractionation, but on the other hand, it is likely that inactivation observed during exposure to temperature can also uptake differentiation factors, which can only be detected with appropriate targeted testing.

The addition of TR solution to the cultivation medium stimulated the production B. subtilis metabolites that suppress the growth of pathogenic bacteria (Fig. 3).

When TR sterilized by filtration was added, the growth of test-strains was completely absent. The autoclaved TR also stimulated the production of antimicrobial metabolites 


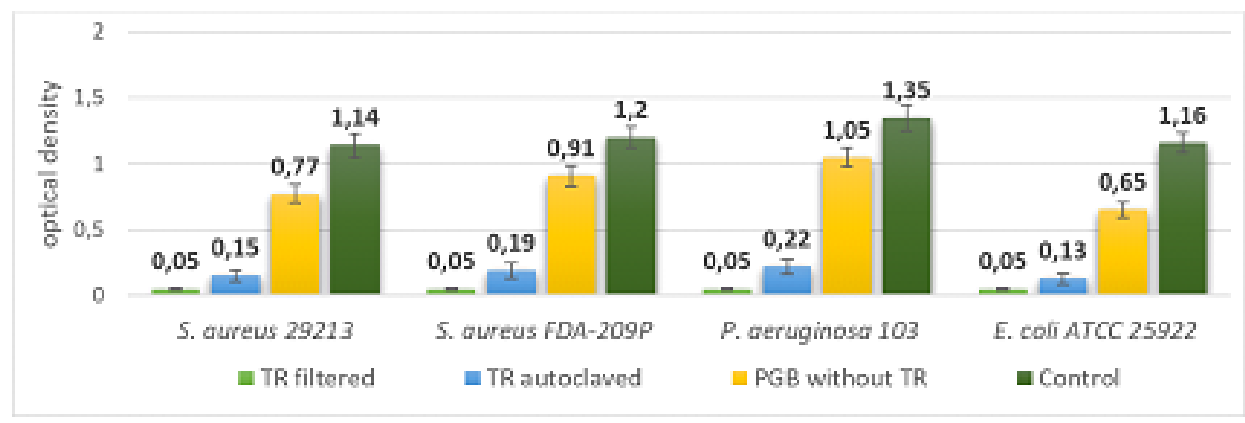

Figure 3: The effect of TR on the antibacterial activity by $B$. subtilis metabolites.

by $B$. subtilis. However, as a result of the experiment was weak growth of test-strains. When $B$. subtilis was cultivated in clean PGB, this property was manifested insignificantly or practically absent.

\section{Conclusion}

The studies carried out indicate that the products of industrial hydrolysis, in addition to nitrogenous nutrients, contain thermostable and thermolabile biologically active substances that affect the growth and functional properties of the $B$. subtilis.

\section{References}

[1] Mikhailova N.A., Grinko O.M. (2010). Bacteria of The Genus Bacillus - Producers of Biologically Active Substances of Antimicrobial action. Journal of Microbiology, Epidemiology and Immunobiology, N.3, P. 85-89

[2] Mikhailova N.A., Voevodin D.A., Lazarev S.A. (2020). Modern ideas about proleukaryotic interactions of the human body are the basis for the creation of a new generation of probiotic drugs. Journal of Microbiology, Epidemiology and Immunobiology, N.4, P. 85-89

[3] Khavinson V.K., Linkova N.S. (2017). United mechanism for peptide regulation of physiological function in living nature. Materials of The XXIII Congress of The Physiological Society by I.P. Pavlov, Istoki, Moscow, P. 1696-1698

[4] Grinko O.M. (2010) Experimental study of the antagonistic properties of the bacterial strain Bacillus Pumillus "Pashkov". PhD Dissertation, Moscow Medical Academy, 2010 